\title{
Age-Related Abnormalities of Circulating Polyamines and Diamine Oxidase Activity in Cystic Fibrosis Heterozygotes and Homozygotes
}

\author{
STEPHEN B. BAYLIN, ${ }^{(25)}$ BERYL J. ROSENSTEIN, LAURENCE J. MARTON, AND \\ DEAN H. LOCKWOOD \\ The Oncology Center (S. B. B.] and the Departments of Medicine I [S. B. B., D. H. L.] and Pediatrics (B. J. R.]. The \\ Johns Hopkins University School of Medicine, Baltimore, Maryland, and The Brain Tumor Research Center. \\ Department of Neurosurgery (L. J. M.] and Department of Laboratory Medicine [L. J. M.], University of California \\ School of Medicine, San Francico, California, USA
}

\begin{abstract}
Summary
Serum putrescine (Pu), spermidine (Sd), spermine (Sp), and plasma diamine oxidase activity (DAO) were determined in cystic fibrosis (CF) homozygotes and heterozygotes plus age-matched controls. Mean ( \pm S.E.) levels of $\mathrm{Pu}(0.51 \pm 0.06 \mathrm{nmoles} / \mathrm{ml})$, Sd $(0.63 \pm 0.07 \mathrm{nmoles} / \mathrm{ml})$, and $\mathrm{Sp}(0.33 \pm 0.08 \mathrm{nmoles} / \mathrm{ml})$ were higher $(P<0.001)$ in 17 control children versus 16 healthy adults $(\mathrm{Pu}=0.15 \pm 0.04 ; \mathrm{Sd}=0.14 \pm 0.03 ; \mathrm{Sp}=0$ in all $)$. All $4 \mathrm{CF}$ adults tested, but only 3 of 24 CF children, had increased poly. amines relative to age-matched controls. $\mathrm{Pu}(0.33 \pm 0.04)$, Sd $(0.33 \pm 0.05)$ and $S p(0.16 \pm 0.12)$ were increased $(P<0.001)$ in 11 adult heterozygotes; in turn, $P u(0.56 \pm 0.11 ; P<0.025)$ and Sd $(0.86 \pm 0.22 ; P<0.005)$ were higher in the adult $C F$ patients than in the heterozygotes. Plasma DAO was higher $(P<0.001)$ in 45 control children $(9.3 \pm 0.7$ units $/ \mathrm{ml})$ versus 61 healthy adults $(5.7 \pm 0.07)$. Levels $\geq+2$ S.D. for controls occurred in $8(21 \%)$ of 39 CF patients. Plasma DAO $(19 \pm 4.8)$ was increased $(P<0.001)$ in 27 adult heterozygotes; $10(37 \%)$ had values $\geq+2$ S.D. for normal adults. One or more polyamines and/or DAO were increased in each of 11 heterozygotes in whom all parameters were measured. These composite abnormalities might be considered as a potential means for identification of asymptomatic CF heterozygotes.
\end{abstract}

\section{Speculation}

Controversy has arisen as to whether an abnormality in polyamine metabolism is present in patients with cystic fibrosis. The present data suggest that altered levels of circulating polyamines do indeed exist and represent a failure of circulating polyamines to decrease with age in individuals homozygous and heterozygous for the cystic fibrosis gene(s). The resulting increase in circulating levels of one or more of the 3 polyamines studied, plus increases in the amine-metabolizing enzyme diamine oxidase, might provide one useful index for identifying adult heterozygotes.

Data from several groups of investigators suggest that an abnormality in the metabolism of polyamines is present in the autosomal recessive disorder, cystic fibrosis. In general, increased levels of putrescine and spermidine and relatively decreased levels of spermine have been found in whole blood and in circulating red blood cells $(8,12,17)$. Controversy exists, however, in that subsequent studies have failed to show differences in whole-blood polyamine concentrations between patients with cystic fibrosis and age-matched control individuals $(7,15)$. In general, the number of patients studied has been small, and the situation regarding polyamine metabolism in heterozygotes for cystic fibrosis is unclear.
If an alteration in polyamine metabolism exists in cystic fibrosis, the mechanism for its occurrence also remains to be defined. Polyamines may be metabolized through enzymatic deamination. Levels of cellular and circulating amine oxidases which are capable of such deamination have not been extensively studied. Activity of benzylamine oxidase, an enzyme present in human tissues which bears similarity to the spermine oxidase of ruminant blood (22), has been found to be normal in the circulation and fibroblasts of patients with cystic fibrosis (17). Polyamines may also be metabolized through conjugation in sites such as the liver; recently, Rosenblum et al. (18) have reported that the in vivo rate of conjugation of $\left[{ }^{14} \mathrm{C}\right]$ spermidine was much depressed in 2 patients with cystic fibrosis.

The present study was undertaken to address two questions. First, the levels of polyamines in the circulation of patients with cystic fibrosis and obligate heterozygotes have been investigated using a sensitive assay system capable of detecting these compounds in serum. Second, circulating diamine oxidase activity (DAO) [EC 1.4.3.6] has been studied in these same individuals. This enzyme activity is another potential means for regulation of polyamine levels in that it catalyzes deamination of putrescine, a diamine product synthesized from the amino acid, ornithine, in the initial and rate-limiting step in polyamine synthesis. The data reveal that cystic fibrosis homozygotes and heterozygotes have abnormal levels of circulating polyamines and DAO activity. A composite profile of these parameters should be considered in the search for biochemical abnormalities which could identify asymptomatic carriers of the cystic fibrosis gene(s).

\section{MATERIALS AND METHODS}

\section{BLOOD SAMPLES}

All plasma and serum samples were obtained by routine venipuncture from patients and control individuals after obtaining informed consent. After routine separation of plasma and serum in a refrigerated centrifuge, these samples were stored at $-20^{\circ} \mathrm{C}$ until time of assay of DAO activity and polyamine levels. The DAO activity is stable in frozen samples for at least 2 months, and the polyamines are stable indefinitely.

\section{PATIENTS}

The age, race, and sex distribution of all of the individuals comprising the present study is shown in Table 1 . The diagnosis of cystic fibrosis was established on the basis of a characteristic clinical picture and a positive quantitative pilocarpine iontophoresis sweat test (chloride concentration $>60 \mathrm{mEq} /$ liter). Shwachman-Kulczycki scores (21) for the patients ranged from 20 to 95 with a mean score of 64.5 (a higher score is indicative of a better 
Table 1. Age, race, and sex distribution of CF patients, CF heterozygotes, and controls

\begin{tabular}{|c|c|c|c|c|c|c|}
\hline \multirow[b]{2}{*}{ Populations } & \multicolumn{2}{|c|}{ Age (yr) } & \multicolumn{2}{|c|}{ Race } & \multicolumn{2}{|c|}{ Sex } \\
\hline & Range & Mean & Black & White & Male & Female \\
\hline Normal children (31) & $2-20$ & 11.6 & 0 & 31 & 20 & 11 \\
\hline CF patients (39) & $2-31^{1}$ & 14.2 & 2 & 37 & 24 & 15 \\
\hline Children with chronic illness (16) & $4-18$ & 10.9 & 5 & 11 & 6 & 10 \\
\hline $\begin{array}{l}\text { Normal adults studied for diamine oxidase ac- } \\
\text { tivity (61) }\end{array}$ & $21-50$ & 27.4 & 3 & 58 & 23 & 38 \\
\hline $\begin{array}{l}\text { Normal adults studied for serum polyamine } \\
\text { levels (16) }\end{array}$ & $20-44$ & 31.8 & 1 & 15 & 9 & 7 \\
\hline CF heterozygotes (27) & $26-56$ & 39.0 & 1 & 26 & 8 & 19 \\
\hline
\end{tabular}

' Four patients range in age from 25 to 31 years old, and the rest are all below age 18.

clinical status). The 27 obligate heterozygotes studied are parents of patients attending the Johns Hopkins Hospital cystic fibrosis clinic. Plasma and serum samples were obtained from the $\mathbf{3 1}$ normal children at the time of a routine office visit and from 16 children with a variety of chronic illnesses, including: asthma (6), immune deficiency disorders with chronic pulmonary disease (4), recurrent pneumonia (2), and diabetes, angioneurotic edema, Wilms' tumor, and rheumatoid arthritis (1 each). The 77 normal adults studied were taking no chronic medications and had no known major medical problems, and none of the individuals were pregnant.

\section{ASSAYS}

Plasma DAO. DAO activity was determined with $\left[{ }^{3} \mathrm{H}\right]$ putrescine as a substrate as previously described in detail $(2,4)$. Results are expressed in units $/ \mathrm{ml}$ plasma where 1 unit $=1$ pmole putrescine deaminated per hr. As in our previous studies (2), we obtained good correlation between results obtained with this method and by the method of Beaven and Jacobsen (6) using $\left[\beta-{ }^{3} \mathrm{H}\right]$ histamine as a substrate. Inasmuch as better discrimination is seen between high and low levels of plasma enzyme activity using the $\left[{ }^{3} \mathrm{H}\right]$ putrescine method (2), the data given in the present study are from this assay system unless otherwise specified.

Serum Polyamine Levels. One $\mathrm{ml}$ of serum was placed in a round-bottom tube and lyophilized; $0.5 \mathrm{ml} 10 \%$ trichloroacetic acid was added to this residue, and the solution was allowed to stand on ice for $30 \mathrm{~min}$ and then centrifuged. The supernatant was transferred into a miniaktor tube (Applied Science), and another $0.5 \mathrm{ml} 10 \%$ trichloroacetic acid was added to the precipitate. The solution was chilled for $30 \mathrm{~min}$ on ice and centrifuged, and the supernatant was added to a miniaktor tube. The combined supernatant was washed twice with excess ether and then lyophilized; the solution was then hydrolyzed by adding $0.5 \mathrm{ml} 6 \mathrm{~N} \mathrm{HCl}$ to the powder, capping the miniaktor tube, and heating at $110^{\circ} \mathrm{C}$ for 14 to $16 \mathrm{hr}$. The hydrolysate was lyophilized, reconstituted in $150 \mu 14 \%$ sulfosalicylic acid, and centrifuged. Fifty $\mu \mathrm{l}$ of the supernatant was used for subsequent chromatographic analysis.

Ion-exchange chromatographic separation of the polyamines with fluorometric detection was carried out on a Durrum D 500 amino acid analyzer (Durrum Instrument Corp., Sunnyvale, CA) as previously described (14) with modifications of the column and buffers. The column was an $11.5 \mathrm{~cm}(1.75 \mathrm{~mm}$ internal diameter) stainless steel column packed with a $16 \%$ cross-linked, $10 \pm 1 \mu \mathrm{m}$, sulfonated polystyrene polymer cation exchange resin (Durrum Chemical Co., Sunnyvale, CA). Four buffers were utilized sequentially for elution of the polyamines from the column. The stock buffer (Buffer IV), used in the last step is $2.40 \mathrm{~N}$ potassium chloride and $0.09 \mathrm{~N}$ potassium citrate ( $\mathrm{pH} \mathrm{5.56)}$ to which thiodiglycol $(12 \mathrm{ml} / \mathrm{liter})$ and liquified phenol $(0.5 \mathrm{ml} / \mathrm{liter})$ had been added. For the first three steps, this is diluted as follows: Buffer I, 1 part stock to 4 parts water; Buffer II, 1 part stock to 1 part water; and Buffer III, 4 parts stock to 1 part water. With this technique, the total recovery for each polyamine averaged $60 \%$; the intraassay coefficient of variation is $7 \%$, and the inter-assay value is $12 \%$.

\section{RESULTS}

\section{SERUM POLYAMINE LEVELS}

Several important relationships are apparent when serum polyamine concentrations are considered for the different age ranges in the subgroups studied (Fig. 1). First, the mean level of each polyamine was significantly higher $(P<0.001$, Student's $t$ test $)$ in the control children than in control adults. No differences between males and females were seen in either group or for the groups of patients and heterozygotes described below. In the children, no statistical differences were observed in mean polyamine levels between those with cystic fibrosis and normal controls. Only one child with cystic fibrosis had an unusually high serum value of spermidine $(>1.2 \mathrm{nmoles} / \mathrm{ml})$, and $3 \mathrm{had}$ high values for spermine $(>1.0 \mathrm{nmoles} / \mathrm{ml})$. However, all 4 adult cystic fibrosis patients (ages 25 to 31 years) had values for each polyamine above the highest value for a normal adult (Fig. 1). As shown by the symbols, for the cystic fibrosis patients, high levels of spermidine in general coincided with high levels of spermine; no such correlation was seen between putrescine levels and the other two amines.

Second and most importantly, the mean levels of putrescine, spermidine, and spermine were elevated in healthy individuals who are heterozygotes for the cystic fibrosis gene(s) as compared to normal adults $(P<0.001)$. Spermine was not measurable by the current technique utilizing $1 \mathrm{ml}$ of serum in any of the 16 normal adults but was measurable (range, 0.06 to 1.32 nmoles/ $\mathrm{ml}$ ) in 7 of 11 cystic fibrosis heterozygotes (Fig. 1). Thus, the polyamine levels in the adult heterozygotes fell midway between those for children (both control and those with cystic fibrosis) and normal adults. In turn, the distribution of values for two of the polyamines, putrescine $(P<0.025)$ and spermidine $(P<0.005)$ was higher in the 4 adult cystic fibrosis patients than that for the heterozygotes. We considered the possibility that the polyamine levels might be lower in the heterozygotes because this group has a higher mean age than for the 4 adult cystic fibrosis patients. However, linear regression analyses of age versus the levels of putrescine and spermidine in the entire normal adult group showed no statistical evidence for a progressive decrease in levels after age 20 . Furthermore, the intermediate position of the heterozygotes between the adult patients and normals is not altered when age is considered as a factor.

\section{PLASMA DAO LEVELS}

The data for plasma DAO activity for all individuals in the study are shown in Figure 2. Despite great overlap between the two groups, the mean value for the 45 control children was significantly higher than that for the 61 control adults $(P<0.001)$. Within the control children, no differences could be found between the 29 normal individuals and the 16 children with various respiratory and other diseases, and as for the polyamine data, this group is hereafter considered as a homogenous one.

The distribution of plasma DAO activity in the patients with cystic fibrosis and in the heterozygotes differed markedly from that for both control children and normal adults. The patients 

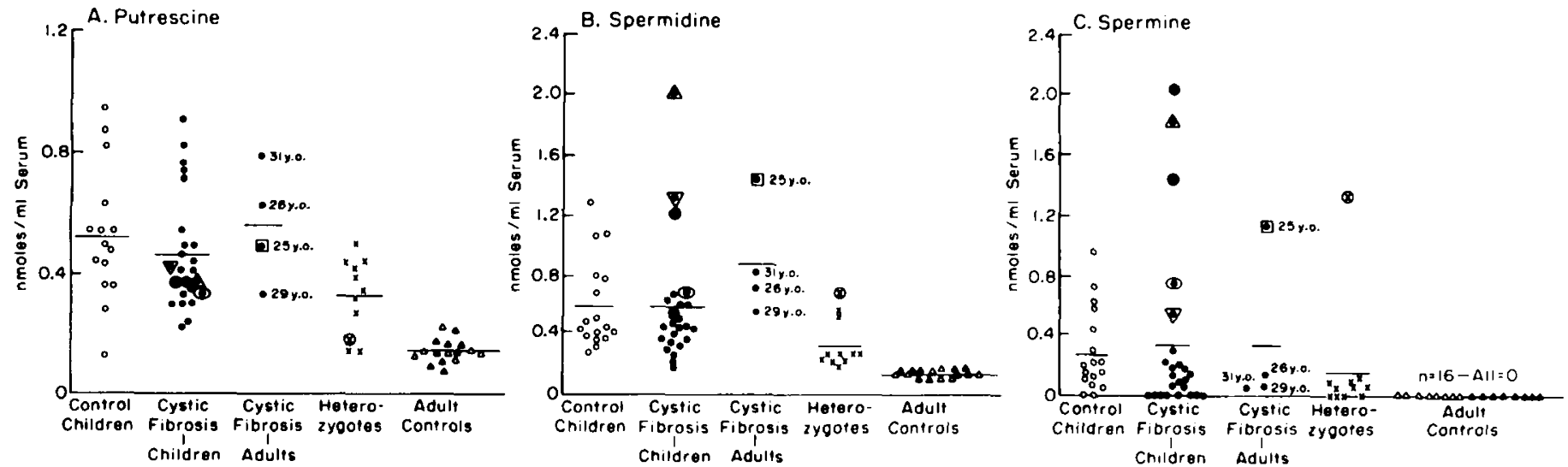

Fig. 1. Serum polyamine levels in control children, patients with cystic fibrosis, obligate heterozygotes for cystic fibrosis, and healthy adults. Individuals were randomly selected from the groups listed in Table 1 for measurement of each polyamine. In the control children group, 8 individuals have chronic illnesses (all chronic pulmonary disorders), and the remainder are healthy individuals; no differences were found between these groups in the control children. The symbols for individuals in the cystic fibrosis groups identify those patients with high spermine values in $C$ so that the relationship between levels of this amine and the other two can be seen. Ages of the adult cystic fibrosis patients are also designated. Patients in the group of children with cystic fibrosis were under age 18. Statistical analyses of the data are given in the text and were obtained by performance of the Student $t$ test. - mean value for group; $\Delta$, male control adult; $\Delta$, female control adult.

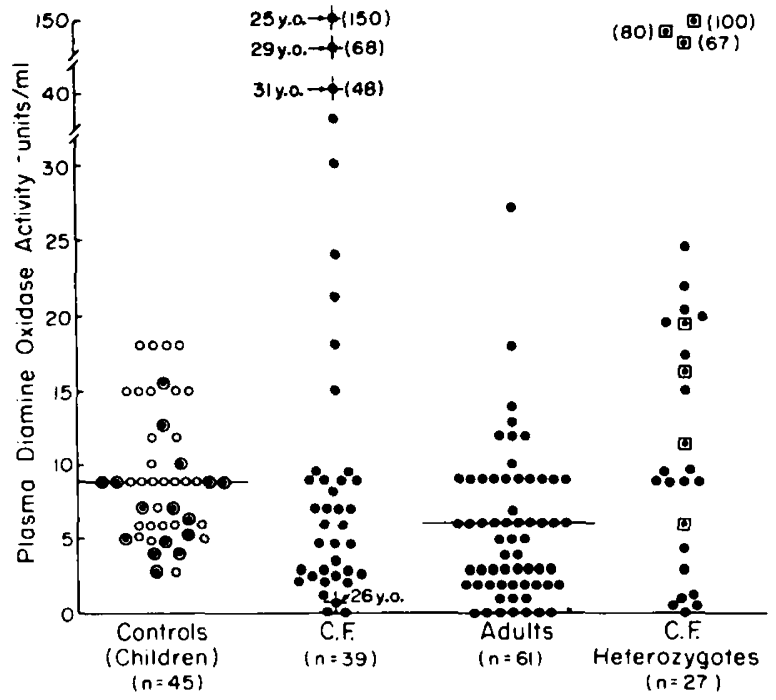

Fig. 2. Plasma diamine oxidase activity in all individuals in the groups studied. $O$, control children with chronic illnesses as detailed in the "Materials and Methods;" $\square$, individuals heterozygous for cystic fibrosis who are parents of patients with plasma levels $\geq 15$ units/ml; ( ), actual values for levels in broken portion of scale. Ages of the adult cystic fibrosis patients are designated.

appear to form two groups; 8 of 39 patients $(21 \%)$ had values equal to or above 18.0 units $/ \mathrm{ml}(+2$ S.D. point for control children), whereas values for all but one of the remainder of the patients were equal to or lower than the mean for control children. The reason for the existence of the two groups is unclear, but age may again be a factor. Three of four adult patients were in the high value group (Fig. 2), and the mean age of the entire 8 patients in this group (19.25 years) was significantly higher $(P<0.05>$ 0.025 ) than that for the other 31 patients ( 12.5 years). In the group of heterozygotes, 10 of 28 tested $(36 \%)$ had values greater than 16.0 units $/ \mathrm{ml}$ ( +2 S.D. point for normal adults); the mean value of 19 units $/ \mathrm{ml}$ for the entire group of heterozygotes differed significantly from that $(6.0$ units $/ \mathrm{ml})$ for the normal adults $(P<$ 0.001 ).

The distribution of DAO activity within families with cystic fibrosis was preliminarily studied. As seen in Figure 2, considerable overlap existed between DAO values in parents of patients who had values above and below 15 units $/ \mathrm{ml}$. However, the mean DAO level of 39 units $/ \mathrm{ml}$ in parents of patients with values $>15$ was higher than that (10 units/ml) for parents of patients with values $<15(P<0.005)$. Also, the mean value of DAO for the parents of patients with low activity is still significantly higher than that for the normal adults $(P<0.005)$. As yet, the number of pairs of parents tested has not been large enough to more precisely define the genetic relationship for DAO activity within families having patients with cystic fibrosis. We also studied 11 families in which 2 siblings with cystic fibrosis were present. Among these sibling sets, the values tended to cluster, and we have found no instance in which one sibling fell into the very high value group and the other into the low group.

\section{HEPARIN STIMULATION OF PLASMA DAO IN PATIENTS WITH CYSTIC FIBROSIS}

Previous studies have shown that plasma DAO increases in normal individuals after IV heparin administration; the rise is proportional to the initial basal level of the enzyme activity $(3,9)$. From animal studies, the source of the increased plasma DAO activity seen after heparin administration is thought to be the gastrointestinal tract $(10,20)$. In the present study, 3 patients with cystic fibrosis were given an IV bolus of heparin at a dose of 10 units/kg. Two patients with high normal initial DAO activities (both, 2.4 units $/ \mathrm{ml}$; mean value for normals, 1.5 units $/ \mathrm{ml}$ ) as measured with the $\left[\beta-{ }^{3} \mathrm{H}\right]$ histamine assay had large increases $(2.1$ and 2.5 units $/ \mathrm{ml})$ whereas one with low basal level $(0.4$ units $/ \mathrm{ml})$ had no increase in plasma DAO activity. The increases for these patients bore the expected relationship to the basal levels when compared to our previously published curves $(3,9)$.

\section{CLINICAL CORRELATIONS}

The patients with cystic fibrosis having DAO values above 15 versus those below 10 units/ml (Figure 2) and those having spermine values above $0.4 \mathrm{nmoles} / \mathrm{ml}$ versus those below (Fig. 1) were compared for the clinical features of their disease. No differences were found for overall Shwachman-Kulczycki scores, types of medications, or incidence of complications such as malabsorption, diabetes, intestinal obstruction, or degree of pulmonary disease.

\section{DIAGNOSTIC SIGNIFICANCE OF POLYAMINE LEVELS AND DAO ACTIVITY IN HETEROZYGOTES}

The composite abnormalities in polyamine levels and DAO activity found in cystic fibrosis heterozygotes were analyzed further (Table 2). Ten of 11 heterozygotes studied had increased serum concentrations of one or more of the three polyamines; the 
Table 2. Polyamine and DAO values for 11 adult heterozygotes

\begin{tabular}{ccccc}
\hline Individual & $\begin{array}{c}\text { Putrescine } \\
\text { (nmoles/ml) }\end{array}$ & $\begin{array}{c}\text { Spermidine } \\
(\text { nmoles/ml) }\end{array}$ & $\begin{array}{c}\text { Spermine } \\
\text { (nmoles/ml) }\end{array}$ & $\begin{array}{c}\text { DAO } \\
\text { (units/ml) }\end{array}$ \\
\hline 1 & $0.44^{1}$ & $0.24^{1}$ & 0 & $19.0^{2}$ \\
2 & $0.44^{1}$ & $0.26^{1}$ & $0.11^{1}$ & 13.6 \\
3 & $0.34^{1}$ & $0.26^{1}$ & $0.07^{1}$ & 8.3 \\
4 & $0.40^{1}$ & $0.25^{1}$ & $0.08^{1}$ & 1.7 \\
5 & $0.50^{1}$ & $0.52^{1}$ & $0.06^{1}$ & $80.0^{1}$ \\
6 & 0.15 & $0.27^{1}$ & 0 & 8.1 \\
7 & $0.42^{1}$ & $0.46^{1}$ & 0 & $63.8^{1}$ \\
8 & $0.27^{1}$ & $0.23^{1}$ & $0.06^{1}$ & 11.0 \\
9 & $0.32^{1}$ & $0.23^{1}$ & $0.08^{1}$ & $100.0^{1}$ \\
10 & 0.15 & $0.18^{2}$ & 0 & $23.4^{2}$ \\
11 & 0.18 & $0.69^{1}$ & $1.32^{1}$ & 9.4 \\
\hline
\end{tabular}

'Value equal to or greater than the highest value seen in age-matched normal adults. Highest values for normal adults: putrescine, 0.23 ; spermidine, 0.19; spermine, 0; DAO. 27.

${ }^{2}$ Values greater than the +2 S.D. value from the mean for normal adults.

eleventh (individual 10) had a spermidine value $>+2$ S.D. from the mean for normal adults. Three of the 11 heterozygotes had an absolute increase in plasma DAO activity; two others had values $>+2$ S.D. from the mean for normals including individual 10 . In summary, all 11 heterozygotes in whom both polyamines and DAO were measured could be differentiated from normal adults on the basis of their possessing abnormalties in one or more of the parameters studied.

\section{DISCUSSION}

The data from our present study further associate an abnormality of polyamine metabolism with cystic fibrosis. The demonstration of elevated levels of circulating DAO activity, an enzyme capable of deaminating putrescine, is a new finding in this disorder. For each of the parameters we studied, the abnormalities became apparent only when age considerations were taken into account. Normal children have significantly higher circulating levels of polyamines and DAO activity than do adults; against this background, the levels in children with cystic fibrosis did not generally differ from those of control children. A distinct abnormality in cystic fibrosis becomes apparent with increasing age. however. The polyamine and DAO activity levels have declined in normal adults whereas patients with cystic fibrosis appear to retain high levels; thus, all four adult cystic fibrosis patients have very high levels of each polyamine as compared to control adults and three of four had high DAO activity. Again, age may have played a role in the bimodal distribution of plasma DAO activity seen in the whole group of cystic fibrosis patients studied.

The abnormalities we describe in circulating polyamine and DAO concentrations may in some manner be related to the cystic fibrosis gene(s) rather than being a secondary manifestation of the disease process. Healthy cystic fibrosis heterozygotes were clearly abnormal in both parameters, and the abnormality in the polyamine levels particularly fits a recessive genetic abnormality. The distribution of serum putrescine and spermidine concentrations in adult heterozygotes, although being distinctly higher than that for adults in a similar age range, was lower than that for the small population of adult cystic fibrosis patients studied. This finding would be expected for a trait in heterozygotes as compared to homozygotes for a recessive gene disorder.

The mechanism underlying the simultaneous abnormalities of circulating polyamines and DAO in patients with cystic fibrosis and heterozygotes is not apparent from the present study. Linear regression analyses of the data (not shown) failed to indicate a significant relationship between the levels of each of the polyamines and the DAO activity. However, certain remarkable analogies are present in the age distribution of these two parameters and in their both being abnormal in the same disease state. Tissue studies may be more revealing in delineating any dynamic link between polyamine metabolism and DAO. Previous data in the rat intestine have revealed a close relationship between levels of $\mathrm{DAO}$ and of ornithine decarboxylase, the enzyme which catalyzes the synthesis of putrescine from ornithine; both enzyme activities increase in parallel with the degree of cellular maturation in the small intestine mucosa (5). Our studies (20) and those of others (10) suggest that much of the circulating DAO may come from intestine in the rat; this may be true for humans as well (9).

In the present study, serum polyamine levels did not distinguish between most patients with cystic fibrosis and control children of a similar age range. These data are in contrast with those of some previous studies in which a high spermidine to spermine ratio has particularly been stressed. One explanation for these differences may lie in the wide range of values which we find for serum spermine concentrations in cystic fibrosis patients. The individuals with very high spermine levels are few and can easily be absent from any given study. Furthermore, previous studies performed have measured circulating polyamine levels in different types of samples. Some have involved the use of whole blood and variations in ratios of the cell types processed could play an important role. Spermine levels as compared to spermidine levels are particularly high in lymphocytes (8), for example, and variations in the yields for these cells could much alter the results obtained. As shown in our data (Fig. 1) when serum is examined, although the majority of children with cystic fibrosis may have low spermine values as compared to the controls, one cannot ignore the wide distribution of values which exist and those patients with quite high spermine levels.

An important potential clinical ramification of the present study is the separation of 11 heterozygotes studied from normal adults by the profile of determinations outlined in Table 2. At present, no laboratory tests have been confirmed which readily detect the cystic fibrosis gene(s) in asymptomatic individuals. The most promising to date is the finding that abnormal circulating levels of a protein with lectin activity causes abnormal agglutination of rat red cells in vitro (11). No one of our measurements provides delineation of the gene(s) carriers from normal. However, our results with the present profile of tests should encourage prospective studies looking at the clinical practicability of applying polyamine and DAO determinations to the identification of individuals heterozygous for the cystic fibrosis gene(s). Although pregnancy and disease states such as neoplasia and psoriasis give rise to increased urinary and circulating polyamine levels $(1,11,13$, $16,19)$ and/or circulating DAO activity $(1,2)$, these are usually readily detectable by clinical means and should not interfere with efforts to identify healthy CF heterozygotes.

\section{REFERENCES AND NOTES}

I. Ahlmark, A.: Studies on the histaminolytic power of plasma with special reference to pregnancy. Acta Physiol. Scand. Suppl., 9: 1 (1944).

2. Baylin, S. B., Abeloff, M. D., Wieman, K. C., Tomford, J. W., and Ettinger. D. S.: Elevated histaminase (diamine oxidase) activity in small-cell carcinoma of the lung. N. Engl. J. Med., 293: 1286 (1975).

3. Baylin, S. B., Beaven, M. A., Krauss, R. M., and Keiser, H. R.: Response of plasma histaminase activity to small doses of heparin in normal subjects and patients with hyperlipoproteinemia. J. Clin. Invest., 52: 1985 (1973).

4. Baylin. S. B., and Margolis, S.: Purification of histaminase (diamine oxidase) from human pregnancy plasma by affinity chromatography. Biochim. Biophys. Acta. 397: 294 (1975).

5. Baylin, S. B., Stevens, S. A., and Shakir, K. M. M.: Association of diamine oxidase and ornithine decarboxylase with maturing cells in rapidly proliferating epithelium. Biochim. Biophys. Acta. 541: 415 (1978)

6. Beaven. M. A., and Jacobsen, S.: A new assay for histaminase activity: measurement of tritiated water from $\beta$ (side-chain label)- ${ }^{3} \mathrm{H}$-histamine. J. Pharmacol. Exp. Ther., 176: 52 (1971).

7. Berry. H. K., Denton. M. D., Glazer, H. S., and Kellogg. F. W.: Normal polyamines in blood of homozygotes for cystic fibrosis. In: R. A. Campbell, D. R. Morris, D. Bartos, G. D. Daves, F. Bartos: Advances in Polyamine Research. Vol. 2. pp. 307-312 (Raven Press, New York, 1978).

8. Cohen, L. F., Lundgren, D. W., and Farrell, P. M.: Distribution of spermidine and spermine in blood from cystic fibrosis patients and control subjects. Blood. 48: 469 (1976).

9. Ettinger, D. S., Baylin, S. B., Minaberry, D., Abeloff, M. D., and Mellits, E. D.: Response of plasma histaminase activity to heparin in normal subjects and in 
patients with small cell carcinoma of the lung. J. Natl. Cancer. Inst., 60: 1239 (1978).

10. Kobayashi, Y., Kupelian, J., and Maudsley, D. V.: Release of diamine oxidase by heparin in the rat. Biochem. Pharmacol., 18: 1585 (1969)

11. Lieberman, J., Costea, N., Jakulis, V., and Kaneshiro, W.: Detection of a lectin in the blood of cystic fibrosis homozygotes and heterozygotes. Clin. Res., 27: S07A (1979).

12. Lundgren. D. W.. Farrell, P. M., and Di Saint'Agnese, P. A.: Polyamine alterations in blood of male homozygotes and heterozygotes for cystic fibrosis. Clin. Chim. Acta, 62: 357 (1975).

13. Marton, L. J., Edwards. M. S., Levin, V. A., Lubich. W. P., and Wilson, C. B.: Predictive value of cerebrospinal fluid polyamines in medulloblastoma. Cancer Res.. 39: 993 (1979).

14. Marton, L. J., and Lee, P. L. Y.: More sensitive automated detection of polya mines in physiological fluids and tissue extracts with $o$-phthaladehyde. Clin. Chem.. 21: 1721 (1975).

15. McEvoy, F. A., and Hartley, C. B.: Polyamines in cystic fibrosis. Pediatr. Res., 9: 721 (1975).

16. Proctor, M., Fletcher, H., Shukla, J., and Rennert, O.: Elevated spermidine and spermine levels in the blood of psoriasis patients. J. Invest. Dermatol., 65: 409 (1975).

17. Rennert O. Frias, J and La Pointe, D: Methylation of RNA and polyamine metabolism in cystic fibrosis. In: J. A. Mangos, R. C. Talamo: Fundamental Problems of Cystic Fibrosis and Related Diseases. pp. 41-52 (Intercontinental Medical Books Corp.. New York. 1973).

18. Rosenblum, M. G. Durie, B. G. M., Beckerman. R. C.. Taussig. L. M., and Russell, D. H.: Cystic fibrosis: decreased conjugation and excretion of $\left[{ }^{14} \mathrm{C}\right]$

Copyright (1) 1980 International Pediatric Research Foundation. Inc. $0031-3998 / 80 / 1408-0921 \$ 02.00 / 0$ spermidine. Science (Wash. D.C.). 200: 1496 (1978)

19. Russell, D. H., Levy, C. C.. Schimpfr, S. C.. and Hawk, I. A.: Urinary polyamines in cancer patients. Cancer Res., 31: 1555 (1971).

20. Shakir, K. M. M., Margolis, S., and Baylin, S. B.: Localization of histaminase (diamine oxidase) in rat small intestinal mucosa: site of release by heparin. Biochem. Pharmacol., 26: 2343 (1977).

21. Shwachman $H$ and Kulczycki, L. I.: Long term study of 105 patients with cystic fibrosis; studies made over a 5-14 year period. Am. J. Dis. Child., 96: 6 (1958).

22. Tabor, H., and Tabor, C. W.: Spermidine, spermine, and related amines. Pharmacol. Rev., 16: 245 (1964)

23. The present address of Dr. D. H. Lockwood is Division of Endocrinology and Metabolism, Department of Medicine. The Rochester University School of Medicine and Dentistry, Rochester. New York 14662

24. The authors are grateful to Kathleen Wieman and Warren Lubich for their expert technical assistance, to Sandra Lund for secretarial assistance, to Dr. Richard C. Talamo for support and constructive criticism. and to Terry Langbaum for data coordination.

25. Requests for reprints should be addressed to: Stephen B. Baylin. M.D., Room 2 127A. The Oncology Center. The Johns Hopkins Medical Institutions, 600 N. Wolfe Street Baltimore, MD 21205 (USA).

26. This research was supported by Grants 5-P50-HL19157. CA-18404, CA-15515. and CA-13525 from NIH and a grant from The National Cystic Fibrosis Foundation. Drs. Baylin (CA-00027) and Marton (CA-00112) are supported by Research Career Development Awards from NIH

27. Received for publication October 9. 1979.

28. Accepted for publication December 13, 1979 